\title{
Retraction Note to: Correlation between PARP-1 Val762Ala polymorphism and the risk of lung cancer in a Chinese population
}

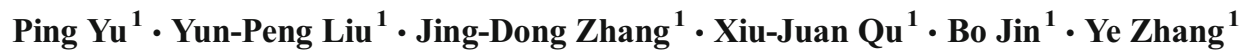

Published online: 19 August 2015

(C) International Society of Oncology and BioMarkers (ISOBM) 2015

Retraction to: Tumor Biol. (2015) 36:177-181

DOI 10.1007/s13277-014-2373-3

The Publisher and Editor retract this article in accordance with the recommendations of the Committee on Publication Ethics (COPE). After a thorough investigation we have strong reason to believe that the peer review process was compromised.

The online version of the original article can be found at http://dx.doi.org/ 10.1007/s13277-014-2373-3.

Yun-Peng Liu

liuyunpeng717@126.com

Department of Medical Oncology, The First Hospital of China, Medical University, Nanjing South Street No. 155, Heping District, Shenyang 110001, People's Republic of China 